\title{
Study of Azo Dye- $n$ TPEB Mixtures in the Nematic and Smectic B Phases by Means of Polarized Light Absorption
}

\author{
Danuta Bauman, Ewa Chrzumnicka, Roman Dąbrowski ${ }^{\mathrm{a}}$, and Jan Jadżyn ${ }^{\mathrm{b}}$ \\ Institute of Physics, Poznań University of Technology, Piotrowo 3, 60-965 Poznań, Poland \\ ${ }^{\text {a }}$ Institute of Chemistry, Military Academy of Technology, Kaliskiego 2, 01-489 Warsaw, Poland \\ b Institute of Molecular Physics, Polish Academy of Sciences, \\ Smoluchowskiego 17, 60-179 Poznań, Poland
}

Z. Naturforsch. 53 a, 813-818 (1998); received June 16, 1998

The influence of the dichroic azo dye on the phase transition temperatures of the mesogenic homologous series of 1-[4- $n$-alkyl-biphenyl]-2-[4-isothio-cyanatophenyl] ethane ( $n$ TPEB) for $n=2-10$ has been examined. For the dye- $n$ TPEB mixtures the polarized absorption spectra as a function of temperature have been recorded. From these spectra the order parameter of the dye dissolved in the liquid crystalline hosts in the whole region of the nematic phase has been determined. The order parameter has revealed the odd-even alternation, similarly as the clearing temperature. In the smectic B phase the mosaic texture has been observed and conclusions concerning structural form of this phase have been drawn.

\section{Introduction}

Materials with liquid crystalline properties are applied in electrooptical displays, optical storage data devices and nonlinear optics. Therefore the synthesis of new substances, which enable the preparation of technologically important mixtures is of interest. Also novel liquid crystalline materials anable to obtain more information about phase transitions, melting behaviour and lowered dimensionality systems, and sometimes lead to exciting surprises.

In most mesogenic substances there exist several liquid crystalline phases between the isotropic and solid phases and on cooling the transition occurs always (except reentrant nematic phase) to the phase with lower symmetry (more ordered). The least ordered is the nematic phase. In this phase the molecules are, on average, aligned with their long axes parallel to each other, what leads macroscopically to a preferred direction, described by the director, $\mathbf{n}$, and the centres of mass of the molecules are placed randomly. Therefore in this phase only a long-range orientational order occurs. In smectic phases the molecular centres of mass sit on planes perpendicular to the preferred axis, leading to a layer structure. In highly ordered

\footnotetext{
Reprint requests to Prof. D. Bauman.
}

smectic B phase the molecules are ordered within the layers with a hexagonal packing and the long axes are perpendicular to the layer. Detailed X-ray diffraction studies of the smectic B modifications have shown that this phase can possess one of two general forms. One structural form (crystal B) is a three-dimensional solid with positional long-range order [ 1 - 4], whereas second one (hexatic B) has only short-range positional ordering of the molecules within the layers. The layers, however, do have a local hexagonal structure and the bond orientational order in the hexatic smectic B phase is long-range $[4,5]$.

In this paper we have studied the homologous series of 1-[4- $n$-alkyl-biphenyl]-2-[4-isothio-cyanatophenyl] ethane ( $n$ TPEB) for $n=2-10$, for which (except $n=2$ and 3) the following phase sequence is observed: crystal - smectic B - nematic - isotropic. Up to now only the dielectric properties in the isotropic phase $[6,7]$ for these substances $(n=6-10)$ and thermal properties under high pressure for 6TPEB [8] have been studied. In our study we have doped $n$ TPEB by dichroic azo dye and, owing to the guesthost effect [9], we have been able to investigate the molecular orientation in the mesophase for the dyeliquid crystal mixtures using polarized light absorption measurements. The influence of the dye on the transition temperatures has also been examined. 
$n$ TPEB

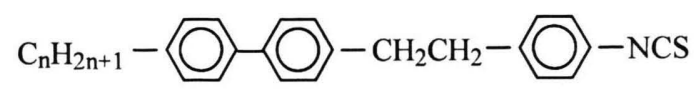

DYE

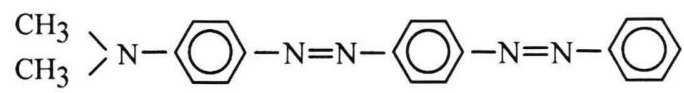

Fig. 1. Molecular structure of the compounds investigated.

\section{Materials and Methods}

The mesogenic 1-[4- $n$-alkyl-biphenyl]-2-[4-isothio-cyanatophenyl] ethanes ( $n$ TPEBs) for $n=2-10$ were synthesized and purified in the Institute of Chemistry at the Military Academy of Technology, Warsaw, Poland. These substances were doped with dichroic azo dye at a concentration of $0.3 \mathrm{wt} \%$. The dye was synthesized and chromatographically purified in the Institute of Dyes at Łódź University of Technology, Poland. Figure 1 presents the chemical formulae of the compounds used.

The textures and phase transitions temperatures, both of pure liquid crystals and of dye-liquid crystal mixtures, were studied with a polarizing microscope JENAPOL (Carl Zeiss Jena, Germany) equipped with a heating stage THMSE 600 (Linkam, UK). The temperature was regulated and controlled with the accuracy of $\pm 0.1^{\circ} \mathrm{C}$ by means of controller TMS 92 (Linkam, UK).

The polarized light absorption of the guest-host mixtures was measured in the visible spectral region with a SPECORD M40 (Carl Zeiss Jena, Germany) spectrophotometer equipped with polarizers. The measurements were made in "sandwich" cells of $20 \mu \mathrm{m}$ thickness. The temperature of the cells was determined with an accuracy of $\pm 0.1^{\circ} \mathrm{C}$ by means of Temperature Controller 660 (Unipan, Poland). The orientation of the guest and host molecules was achieved by treatment of the glass surfaces of the cells with polyimide and by an additional rubbing process. This procedure gives a homogeneous planar molecular orientation in the nematic phase, that was controlled with the aid of crossed polarizers. The absorption spectra of the dye dissolved in the liquid crystals were recorded for two positions of the cell, that is with the orientation of the liquid crystal at angles of $0^{\circ}$ and $90^{\circ}$ with respect to the polarization plane of the light beam. Additionally, the base line spectra for the pure liquid crystals were recorded for the same two positions of the cell. After correction for the base line, the two components of the absorbance were obtained as functions of the wavelength.

The long-range orientational order which is characteristic of liquid crystals in the uniaxial phases, is usually described by the order parameter [10]

$$
S=\frac{1}{2}\left(3\left\langle\cos ^{2} \theta\right\rangle-1\right),
$$

where $\theta$ is the angle between the long molecular axis and the optical axis of the liquid crystal.

The order parameter of the guest aligned in the liquid crystalline host can also be determined from (1), but in this case $\theta$ is the angle between the long axis of the guest molecule and the direction of the host orientation. If guest molecules are chosen such that their geometric long axis corresponds to the direction of the absorption polarization and if the contribution to the absorption caused by molecular polarization in the perpendicular direction is small, then the order parameter can be obtained directly from the spectrophotometric data. The azo dye used in this study fulfils these requirements: the molecule is cylindrically shaped and the direction of the charge transfer absorption transition, which gives rise to its colour, is parallel to the long molecular axis. Therefore, $S$ can be determined by using the equation $[11,12]$

$$
S=\frac{A_{\|}-A_{\perp}}{A_{\|}+2 A_{\perp}}
$$

where $A_{\|}$and $A_{\perp}$ are the absorbances of the dye in the host medium when the electric vector of the incident light is, respectively, parallel and perpendicular to the orientation axis of the liquid crystal.

\section{Results and Discussion}

\section{III.1. Phase Transitions Temperatures}

Table 1 gathers the temperatures of the phase transitions observed for homologous series of $n$ TPEB. The melting temperature was determined by heating the sample, the temperature of the transition between liquid crystalline phases was determined by cooling 
Table 1. Phase transition temperatures (in ${ }^{\circ} \mathrm{C}$ ) for homologous series of $n$ TPEB; brackets indicate a monotropic transition.

\begin{tabular}{crcl}
\hline$n$ & $\mathrm{C}-\mathrm{S}_{\mathrm{B}}$ & $\mathrm{S}_{\mathrm{B}}-\mathrm{N}$ & $\mathrm{N}-\mathrm{I}$ \\
\hline 2 & 129.2 & - & 136.4 \\
3 & 112.0 & - & 145.5 \\
4 & 87.5 & $(88.7)$ & 132.6 \\
5 & 97.0 & $(98.3)$ & 142.6 \\
6 & 56.5 & 100.5 & 132.6 \\
7 & 70.0 & 103.3 & 135.2 \\
8 & 57.0 & 106.3 & 130.3 \\
9 & 68.0 & 106.1 & 130.2 \\
10 & 43.0 & 105.4 & 127.6 \\
\hline
\end{tabular}

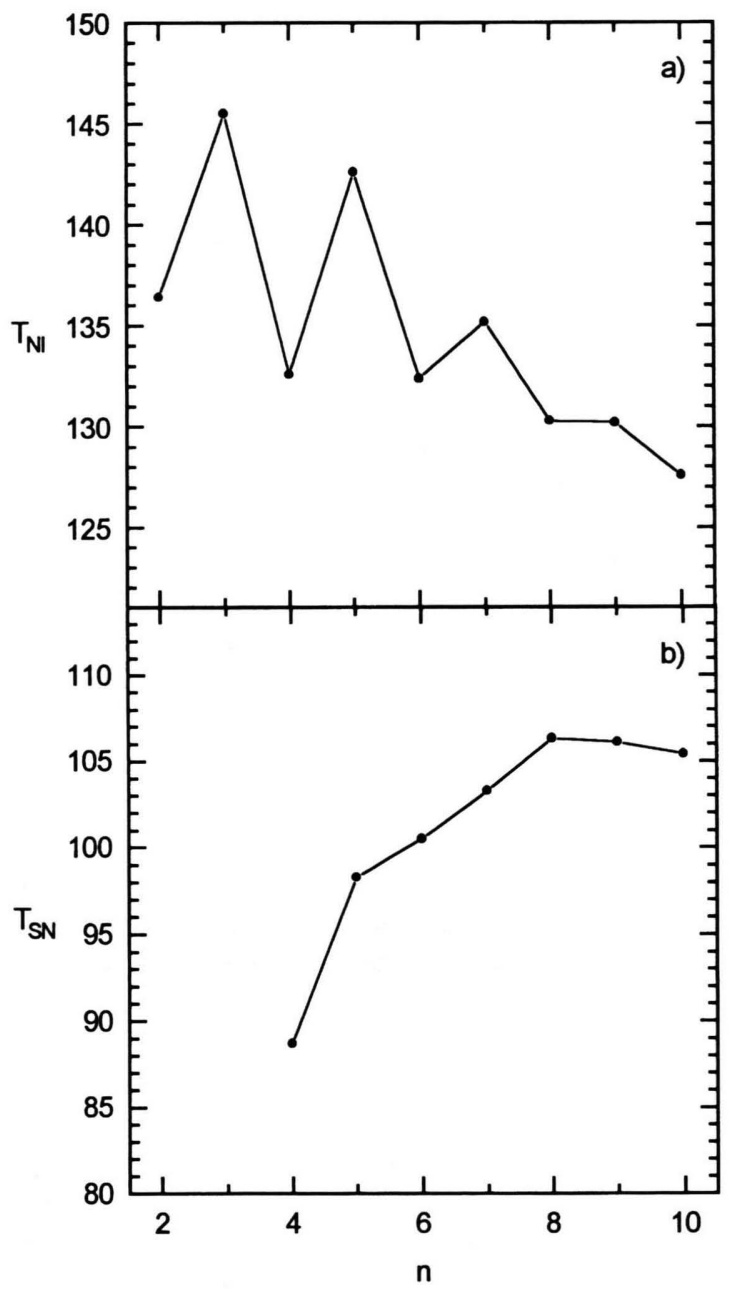

Fig. 2. Phase transition temperatures for $n$ TPEBs as a function of carbon atom number: a) clearing temperature $\left(T_{\mathrm{NI}}\right)$, b) nematic-smectic B transition temperature $\left(T_{\mathrm{SN}}\right)$. the sample, whereas the clearing temperature is the average of the temperatures obtained by heating and cooling (the difference was not greater than $\pm 0.2^{\circ} \mathrm{C}$ ). 2TPEB and 3TPEB have only one mesogenic (nematic) phase, whereas for 4TPEB and 5TPEB a strong supercooling effect is observed, and before the transition to the solid state, the smectic B phase appears after the nematic one. For $n=6-10$ two enantiotropic liquid crystalline phases occur. From the results presented in Table 1 it is seen that with the increase of $n$ a tendency of narrowing the nematic phase range occurs. The estimation of the range of the smectic $B$ phase is difficult in the case of the occurrence of the monotropic phase, but one can notice that for $n=10$ the smectic B phase occupies the widest temperature range.

It is well known, that the nematic-isotropic transition temperature, $T_{\mathrm{NI}}$ for the various homologous series of liquid crystals shows the odd-even effect [13]: for even members of the series, $T_{\mathrm{NI}}$ is always smaller than for the previous odd member. Such a behaviour had been explained as the alternation of the interaction between alkyl chains [14]. Looking at the results of $T_{\mathrm{NI}}$ listed in Table 1 and presented additionally in Fig. 2a for $n$ TPEBs, it is seen that this homologous series shows the odd-even effect for $T_{\mathrm{NI}}$ very distinctly. Moreover, as it has been predicted by theory for series with higher transition temperatures $[14,15]$, a decrease of $T_{\mathrm{NI}}$ with $n$ is observed. As the alkyl chain becomes longer, due to its flexibility the odd-even alternation decays progressively.

The nematic-smectic B transition temperature does not show the odd-even effect (Fig. 2b), but a trend to an increase of this temperature is observed.

It had been found [16 - 20] that the addition of a dichroic dye to a nematogenic liquid crystal changes the nematic-isotropic transition temperature, causing either a decrease or an increase of the clearing point with respect to that of a pure host. Moreover, it had been ascertained [17-20], that the presence of the elongated dye molecules in the nematic matrix leads to the formation of a two-phase region in the vicinity of the clearing point, in which nematic and isotropic phases coexist in equilibrium. The azo dye used in this study is added to the liquid crystals at such small concentration that the two-phase region cannot be registered: if it occurs, it must be narrower than $0.1^{\circ} \mathrm{C}$. However, the changes of $T_{\mathrm{NI}}$ are observed. Figure $3 \mathrm{a}$ presents the shift of the nematic-isotropic transition temperature $\left(\Delta \mathrm{T}_{\mathrm{NI}}\right)$ for $n$ TPEBs after addition of the 


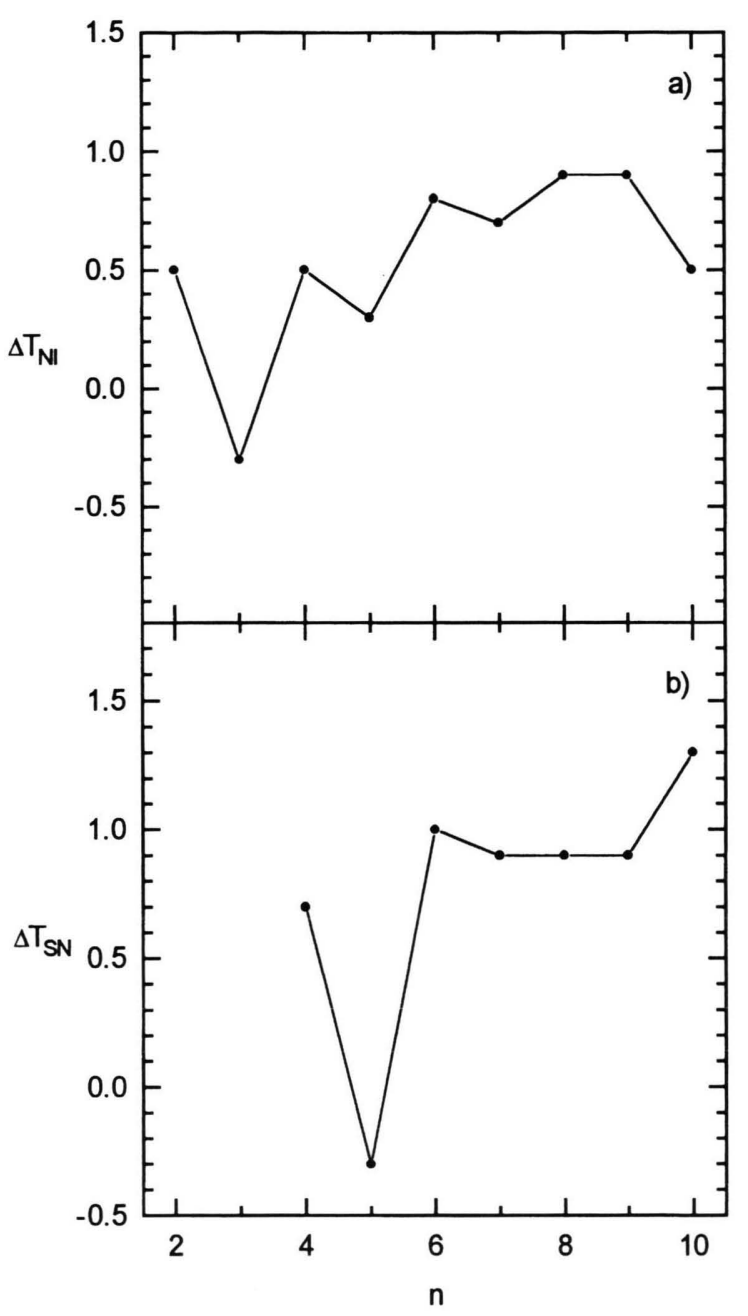

Fig. 3. Shift of: a) nematic-isotropic transition temperature $\left(\Delta T_{\mathrm{NI}}\right)$ and b) nematic-smectic $\mathrm{B}$ transition temperature $\left(\Delta T_{\mathrm{SN}}\right)$ for $n$ TPEBs after azo dye addition $(c=0.3 \% \mathrm{wt})$.

dye. It is seen that except 3TPEB, this shift is positive and reveals the odd-even effect. It is interesting to notice that a greater increase of $T_{\mathrm{NI}}$ occurs for even member of the $n$ TPEB series, than for previous odd member (except for $n=9,10$ ). This is probably connected with the influence of a dye on the order parameter of a liquid crystalline host [17], which will be discussed in detail in the next section.

The presence of the dye in the liquid crystals investigated influences also the temperature of the transition from the nematic to the smectic B phase (Fig. 3b), but no regular behaviour is observed.

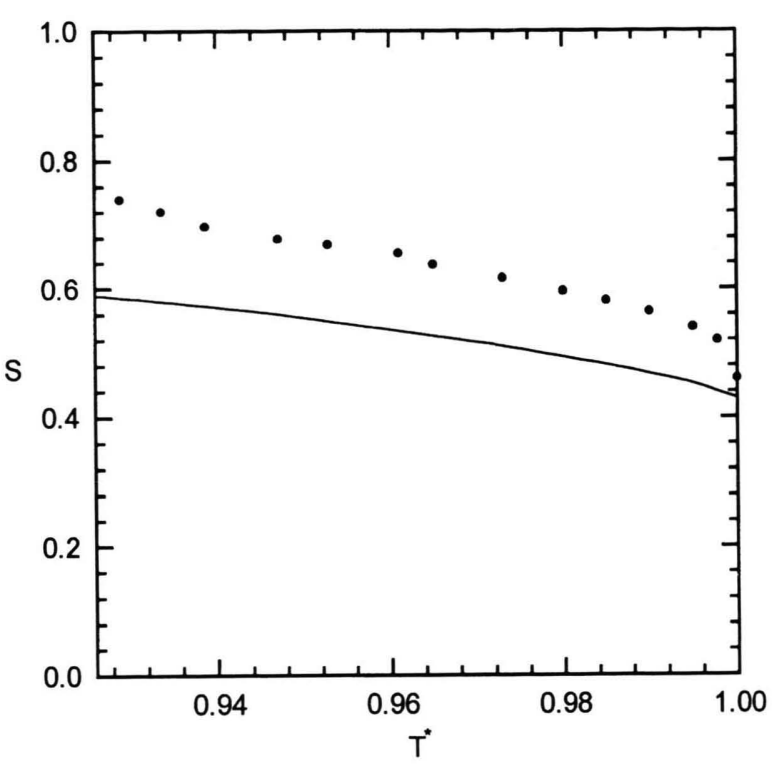

Fig. 4. Order parameter $S$ versus reduced temperature for dye-6TPEB mixture. Solid line: Maier-Saupe theory prediction [25].

\section{III.2. Order Parameter of the Dye-Liquid Crystal Mixtures}

Figure 4 shows the dependence of the order parameter $\mathrm{S}$ on the reduced temperature $T^{*}=T / T_{\mathrm{NI}}$ for 6TPEB as an example, whereas in Fig. 5 the values of the order parameter at two different temperatures for the homologous series investigated are presented. The order parameter was calculated from (2), where as $A_{\|}$and $A_{\perp}$ the values of the absorbances at the wavelength corresponding to the maximum of the dye absorption $\left(\lambda_{\max }=496 \mathrm{~nm}\right)$ were taken. Only the results for the nematic phase are presented in Figs. 4 and 5.

It had been previously found [17, 20 - 22] that the dyes which cause an increase of the clearing temperature of the liquid crystalline host have a good compatibility with the nematic phase, i.e. the correlation between the mutual parallel alignment of the dye and liquid crystal molecular long axes is very strong. In such a case the order parameter of the dye is at least so high as the order parameter of the host, and sometimes it is even significantly higher, especially if $\Delta T_{\mathrm{NI}}$ is large [22]. This effect is, of course, connected also with the relation between the sizes of the guest and host molecules. Computer calculations made with the 


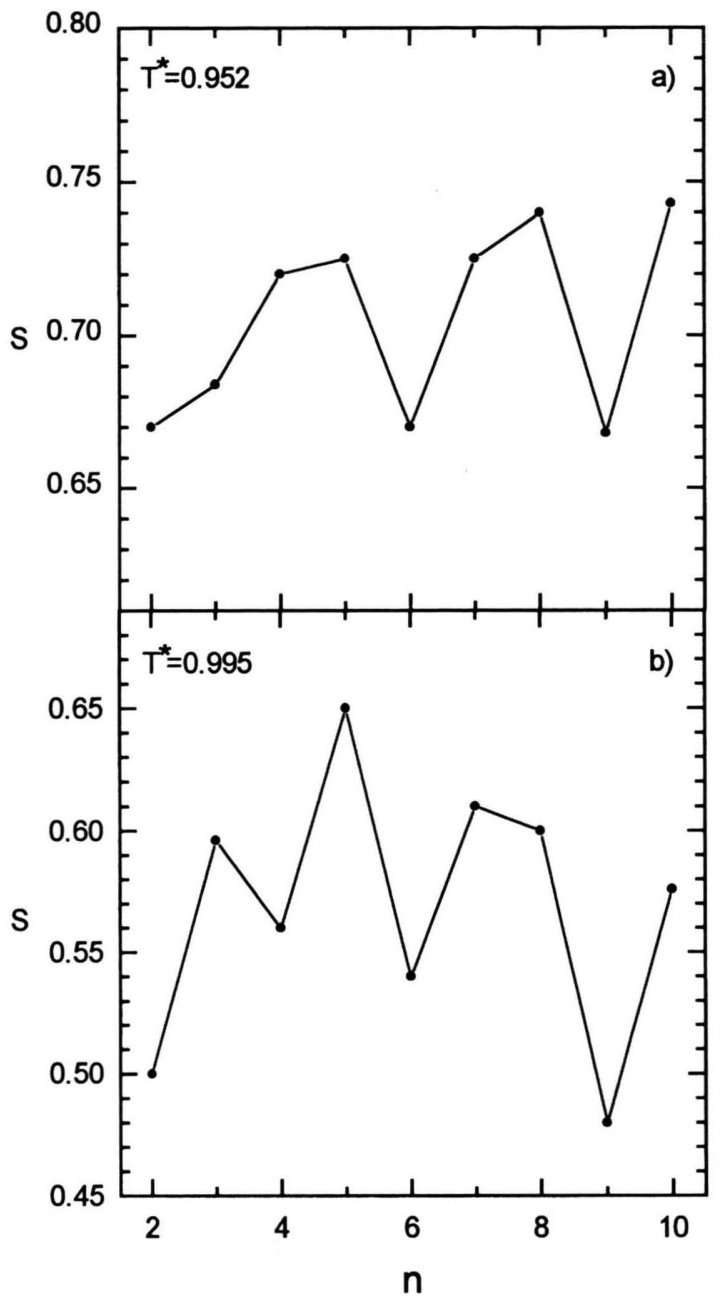

Fig. 5. Order parameter, $S$ for dye- $n$ TPEB mixtures as a function of carbon atom number at two different reduced temperatures.

HyperChem program have indicated that the length of the azo dye molecule used in this study is about $20 \AA$. The length of the $n$ TPEB molecules is similar: for 3TPEB it is about $22 \AA$. Taking into account the flexibility of the alkyl chain one can assume that the increase of $n$ does not cause the rise of the molecule length in the $n$ TPEB series significantly $[21,23,24]$. As the dye added to the liquid crystals (except 3TPEB) increases their nematic-isotropic transition temperature, it is reasonable to suppose that the order parameter of the dye reflects very well the molecular orientation of the nematic host itself. The results presented in Figs. 4 and 5 indicate that all members of the homologous series of $n$ TPEB are characterized

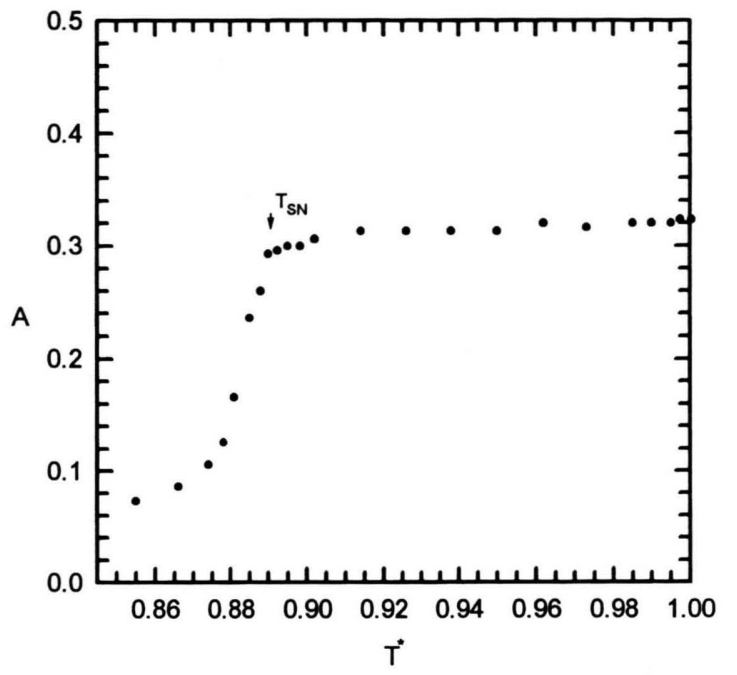

Fig. 6. Absorption of dye-5TPEB mixture versus reduced temperature in the smectic B and nematic phases.

by a very high order parameter in the whole range of the nematic phase: only for 9TPEB at $T_{\mathrm{NI}}$ the order parameter drops somewhat below 0.5 . In the vicinity of the transition to the smectic B phase (or crystal state in the case of 2TPEB and 3TPEB) $S$ reaches the value between 0.67 and 0.74 , depending on the liquid crystalline host. Such values of $S$ obtained for the $n$ TPEB series are significantly higher than those predicted by the Maier-Saupe theory [25] (Fig. 4) and those determined for many other liquid crystals [17, $21,22,26-31]$. This effect can be connected with the length of $n$ TPEB molecules (they have three benzene rings, whereas other liquid crystals investigated possess only two aromatic rings) and/or with the occurrence of the very highly ordered smectic B phase.

Looking at the data presented in Fig. 5, it is seen that the character of the changes of the order parameter with the number of carbon atom is different at various reduced temperatures. This means that the liquid crystals under investigation are very differently resistant to thermal fluctuations. In the vicinity of the nematic-isotropic phase transition the odd-even effect occurs: for odd members of the series the value of $S$ is higher than for the previous even member. This is very well correlated with the values of the clearing temperature. The odd-even alternation vanishes for $n=9$, similarly as it has been observed for $T_{\mathrm{NI}}$.

Because the smectic B phase is also a uniaxial one, we were going to determine the order parameter in this phase using the same method as for the nematic phase. 
Unfortunately, the absorption measurements have revealed that the total absorbance, calculated from the relation $A=\left(A_{\|}+2 A_{\perp}\right) / 3$, decreases rapidly on cooling from the nematic phase. This is illustrated in Fig. 6 for 5TPEB as an example. The observations under the polarizing microscope have shown that at the temperature corresponding to the drop of the absorption, the molecules cease from keeping the homogeneous planar orientation. The phase which has been formed on cooling the nematic phase has exhibited a mosaic texture, which is one of the two naturally occurring textures of the smectic B phase [32]. In the mosaic area some regions occur, in which the smectic layer planes are planar to the surfaces, which means that the molecules are aligned homeotropically. This can cause the diminishing of the dye absorption. Moreover, the observations under the microscope have revealed that the dye and the liquid crystal do not create longer a homogenous mixture: precipitation of

[1] A. J. Leadbetter, M. A. Mazid, B. A. Kelly, J. W. Goodby, and G. W. Gray, Phys. Rev. Lett. 43, 630 (1979).

[2] D. E. Moncton and R. Pindak, Phys. Rev. Lett. 43, 701 (1979).

[3] P. S. Pershan, G. Aeppli, J. D. Litster, and R. J. Birgenean, Mol. Cryst. Liq. Cryst. 67, 205 (1981).

[4] J. W. Goodby and R. Pindak, Mol. Cryst. Liq. Cryst. 75, 233 (1981).

[5] R. Pindak, D. E. Moncton, S. C. Davy, and J. W. Goodby, Phys. Rev. Lett. 46, 1135 (1981).

[6] J. Jadżyn, G. Czechowski, B. Żywucki, C. Legrand, P. Bonnet, and R. Dąbrowski, Z. Naturforsch. 48a, 871 (1993).

[7] J. Jadżyn, C. Legrand, N. Isaert, A. Cartier, P. Bonnet, G. Czechowski, and B. Żywucki, J. Mol. Liq. 62, 55 (1994).

[8] C. Ernst, G. M. Schneider, A. Würflinger, J. Jadżyn, and R. Dąbrowski, Z. Naturforsch. 62a, 490 (1997).

[9] G. H. Heilmeier and L. A. Zanoni, Appl. Phys. 13, 91 (1968).

[10] V. Zwetkoff, Acta Phys. USSR, 10, 557 (1939).

[11] V. G. Rumyantsev, L. M. Blinov, and V. A. Kizel, Kristallografiya 18, 1101 (1973).

[12] D. L. White and G. N. Taylor, J. Appl. Phys. 45, 4718 (1974).

[13] "Flüssige Kristalle in Tabellen II", VEB Deutscher Verlag für Grundstoffindustrie, Leipzig 1984.

[14] S. Marčelja, J. Chem. Phys. 60, 3599 (1974).

[15] W. H. de Jeu, J. van der Veen, and W. J. A. Goossens, Sol. State Commun. 12, 405 (1973).

[16] W. Haase, O. Trinquet, U. Quotschalla, and J. Foitzik, Mol. Cryst. Liq. Cryst. 148, 15 (1987).

[17] D. Bauman, Mol. Cryst. Liq. Cryst. 159, 197 (1988).

[18] D. Bauman, Z.-X. Fan, and W. Haase, Acta Phys. Pol. A 80, 545 (1991).

[19] D. Bauman, H. Moryson, and J. Jadżyn, Acta Phys. Pol. A 85, 553 (1994). the dye has been observed. This fact can be considered as a second reason of the absorption decrease, and simultaneously allows to suppose that the structural form of the smectic B phase for $n$ TPEBs is crystallike. The results of the preliminary dielectric relaxation studies for 10TPEB [33] seem to confirm this statement. According to the classification proposed by Kresse and Gajewska [34] on the basis of the ratio of the relaxation times connected with the molecules rotation around their short axis in the smectic B and nematic phases, one is able to ascertain which structural form of smectic B phase occurs. For 10TPEB the $\tau_{1}\left(\mathrm{~S}_{\mathrm{B}}\right) / \tau_{2}(\mathrm{~N})$ value of about 10 had been obtained, what could mean that the smectic B phase of $n$ TPEBs is a crystal-like modification.

\section{Acknowledgements}

This work was supported by Poznan University of Technology Research Project No TB 62-147/98-DS.

[20] D. Bauman, J. Solczyński, and E. Chrzumnicka, Acta Phys. Pol. A 92, 1151 (1997).

[21] D. Bauman, Mol. Cryst. Liq. Cryst. 172, 41 (1989).

[22] D. Bauman and H. Moryson, J. Mol. Structure 404, 113 (1997).

[23] H. Seki, T. Uchida, and Y. Shibata, Mol. Cryst. Liq. Cryst. 138, 349 (1986).

[24] A. S. Paranjpe, K. U. Deniz, P. S. Parvathanathan, V. Arnithalingan, and K. V. Muralidharan, Mol. Cryst. Liq. Cryst. 149, 79 (1987).

[25] W. Maier and A. Saupe, Z. Naturforsch. 14a, 882 (1959); 15a, 287 (1960).

[26] R. Seeliger, H. Haspeklo, and F. Noack, Mol. Phys. 49, 1039 (1983).

[27] L. G. P. Dalmolen, S. J. Picken, A. F. de Jong, and W. H. de Jeu, J. Phys. (Paris) 46, 1443 (1985).

[28] M. Mitra, Mol. Cryst. Liq. Cryst. 241, 17 (1994).

[29] B. Żywucki, W. Kuczyński, and G. Czechowski, in: Liquid Crystals: Materials Science and Applications, J. Żmija, Z. Raszewski, J. Zieliński, eds., Proc. SPIE, 2372, 151 (1995).

[30] G. Czechowski, B. Żywucki, D. Bauman, and J. Jadżyn, in: Liquid Crystals: Materials Science and Applications, J. Żmija, Z. Raszewski, J. Zieliński, eds., Proc. SPIE, 2372, 163 (1995).

[31] H. Herba, B. Żywucki, G. Czechowski, D. Bauman, A. Wąsik, and J. Jadżyn, Acta Phys. Pol. A 87, 985 (1995).

[32] G. W. Gray and J. W. Goodby, Smectic Liquid Crystals, L. Hill, Glasgow 1984.

[33] J. Jadżyn and C. Legrand, unpublished results.

[34] H. Kresse and B. Gajewska, Crystal Res. Technol. 18, 281 (1983). 